\title{
A Modified Digital Image Processing Enabled Stroke Rehabilitation System based on Wavelet Transform
}

\author{
Rajeshkannan S, Kerthana R
}

\begin{abstract}
: stroke rehabilitation therapy is for people suffering from paralysis. Regular procedures need the involvement of analyst for the duration of the plenary, requires excessive amount. Recently, many approaches have been proposed with control strategy through gesture recognition. The main objective of this work is hand gesture control for stroke rehabilitation based on dwt based feature extraction method. Establishment of artificial machine based computer system assistance provides instruction for paralysis recovery system. The proposed system uses reverse biorthogonal wavelet with two-level decomposition. Preprocessing and image segmentation of real time movement of the hand gestures. Feature extraction is done using discrete wavelet transform (dwt) based approach which has very flexible and adaptable even at the cost of imperfect reconstruction. The prosthetic based robotic hand for human finger movement recovery function. Human being finger movement process helps to rotate motors packed inside the robotic finger section. Therefore, the five finger machine finger movement can imitate the real time human being gesture in a regular manner, which indicates the newly modified machine represents as a education device to provide recovery for the persons affected after paralysis.
\end{abstract}

Key words: discrete wavelet transform, paralysis, robot, stroke.

\section{INTRODUCTION}

Interplay between normal person and digital device has enhanced a necessary part in day to day scenario. It is concerned with the analysis, appraisal and enactment of interplay digital devices for person use. The general and common gadgets that are used for communication are keyboard, mice, light pen, trackball, keypads etc. These gadgets are popular but not recommended for transmission. Due to the advancement of computer-based interconnection, now computers are able to watch, which makes the mortal and digital device interprocess rich. This employs an advanced transmission and managing facility that is more robust, amicable and time preserving. Plenty of analysis.Process has been made on finger movement gesticulation identification related on various process and methodologies.

Revised Manuscript Received on October 12, 2019

Rajeshkannan Sundararajan, Associate Professor, Department of Electronics and communication, St. Joseph's College of Engineering, Chennai, India.

R.kerthana, B.E degree in Electronics and Instrumentation Engineering from Jeppiaar Engineering College, Chennai, India
The main purpose of using gesticulation need to provide a simpler way of managing and offers a prosperous and creative form of interprocess with the robot based system. This currently facilitates Imaging operations and computer based learning for the device or accomplishment growth. Relating to the World Health Organization (WHO), paralysis is the important and superior reason of demise in the entire globe. About 65.5 trillion rates of deaths worldwide in $2016,10 \%$ of them were due to paralysis and resulting for 6.24 million deaths Stroke is additionally a important purpose of chronic severances. Up to about $90 \%$ of paralysis healers, ensuing in dilemma in top limb characteristic. Most of the treating methods are mankind intensive and require regular meeting with analyst. As a result, the patients affected from paralysis need to use their time in spending around the analyst. Although regular evaluating process has been started and assessment answers are supported generally, there are still occurrence of some drawbacks such as lack of knowledge, shortfalls of mankind and consumption of time. Rehabilitation is the integral approach for top limb feature recovery. Interaction between human and laptop has turn out to be an essential part in day to day life. It is worried with the analysis, contrast and enforcement of inter-connecting digital systems for mankind. The superior famous gadgets that are used for verbal exchange are different computer based devices etc. These devices are popular but not so regular for communication. Due to the growth of computer-based interface, now digital devices are able to watch, which makes the person and machine communication rich. This employs a new transmission and processing facility that is more robust, amicable and consumption of time. Plenty of analysis has been done on hand gesticulation identification based on various methodologies and analysis. The main purpose of using gesticulations is to provide a more common way of managing and facilitate creative way of intercommunication with the digitally developed robot machine. This primarily involves Imaging methods and computer vision for the process or specific growth.

\section{IMPLEMENTATION}

\section{A. Existing Model}

Many processes available that are needed for managing the automatic machine through gesticulations. Some gesticulation focus structures facilitates related shade segmentation, finger movement finding and labeling with compression, noise filtering, and then gesticulations process are 
determined through similarity identification and contouring. This does not facilitate versatility for the gesticulation inputs due to similarity identification. Some systems uses computer intercommunication device to provide real-time gesticulations to the automatic machine. Analog variable resistors which are known as flex sensors are used on the human beings forearm glove to measure the finger bending, also hand placement and are detected by ultrasonic's for gesticulation recognition. And in different method, gesticulations are identified using Microsoft Xbox 360 Kinect(C). It collects the brightness movement section provides the customers the remarks of forearm based information to do and provide them fortify their movement methods. The disadvantages in existing model are use of smart arm band which is of

\section{B. Proposed Model}

In the recent years, many scholars experimented of hand gesticulation identification for mankind pc interplay providing MATLAB and openCV. The overall results comparisons are performed to enhance the method. Here, the human finger movement gesticulations are recognized in MATLAB. The system proposed here uses image processing technique for hand gesture recognition. This image processing technique happens in 3 steps. They are: Capture, comparison and signaling. Usually the capturing process can be done by the webcam of the system. The image thus captured is then converted to black and white image and is then compared with the background image to extract the hand gesture alone. In the proposed system, the gestures are specifically for stroke rehabilitation system. Based on the specific hand gesture is analyzed and is send via wireless module to the Robot. The robot movement is actually controlled by the polarity of the motor which varies as per the signal send by the ZigBee module. Imaging operations are performed out on the RGB picture to change into gray-scale picture. The grayscale picture changed into Black and white picture. This flow process requires similar and plane background. Contouring detection algorithm is used to identify the corners in the picture. By utilizing purpose of edge detection the projection of fist is identified. The feature extraction is based on DWT (DISCRETE WAVELET TRANSFORM). This method is very flexible and adaptable even at the cost of imperfect reconstruction. Features such as histogram of gradients(hog),local binary pattern(lbp),Entropy, entropy filtering, range filtering, gray level co-occurrence matrix,mean,properties of image regions(area,perimeter,eccentricity,orientation,solidity).T he robotic finger section can emulate the user's gesture in a natural way. Hence the new modified system can be served as a practicing machine to support recovery system for the healers after paralysis. A prosthetics based robotic finger section dexterous finger movement moved by computer based methods was implemented for recovery from paralysis. The flow diagram of rehabilitation model is shown in Fig. 1. and depth content using an RGB and Infra-Red camera. This process though is not very reduced rate. A paralysis recovery process has been enforced, involves an effective handy armband, computer vision algorithms, and a prosthetics based robotic finger section. The handy and usable armband is not cost effective. Utilization of CCEA and PCA algorithms. A prosthetics based robotic finger hand moved by computer based algorithm was developed for paralysis recovery. The natural results help from the robotic

finger

high cost. Feature extraction method does not fully give the exact features of hand gestures. Hence the features were extracted with some modification in proposed model.

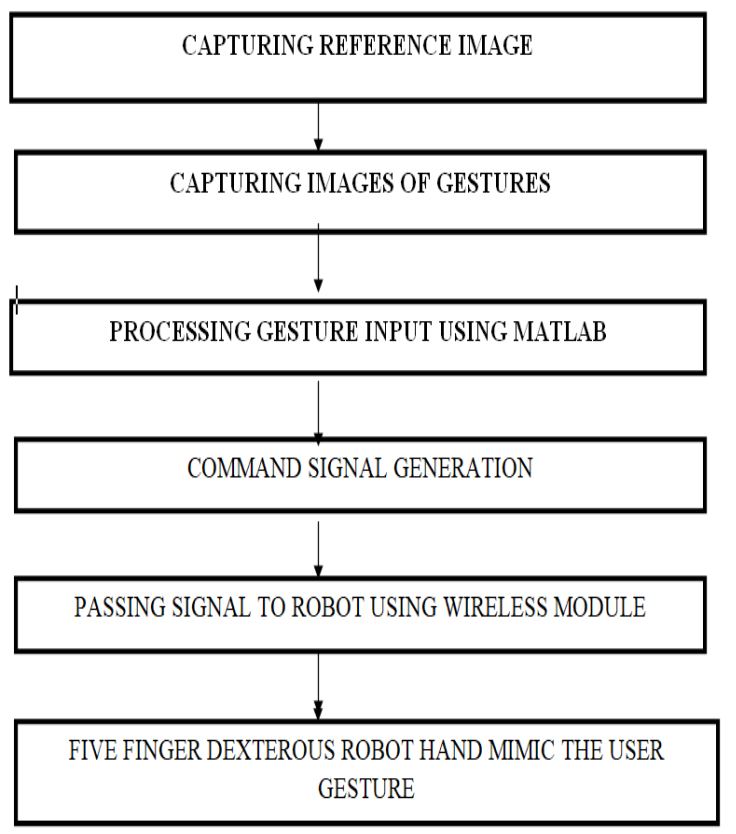

Fig. 1. Flow diagram of modified stroke rehabilitation system

\section{Block Diagram of Modified Stroke Rehabilitation System Description}

The sequenced modified method involves of human being gesticulation collection of pictures, enhancement of those pictures, differentiation into segments of those pictures using edge detection process, extracting versatile details of hand using DWT process. The Block diagram of our proposed rehabilitation model is shown in Fig. 2 and steps involved are given below. 


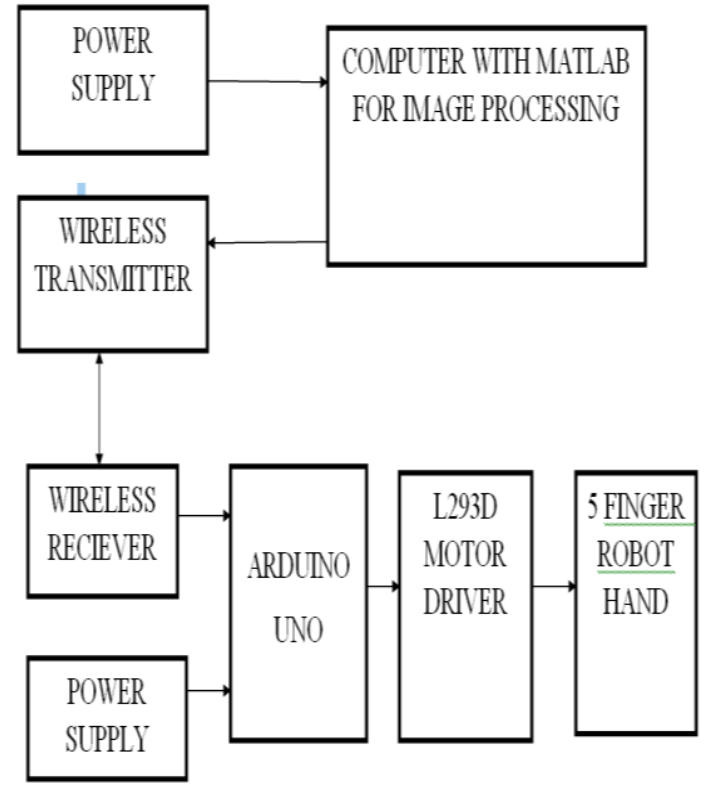
system

\section{C.1 Image Acquisition}

Picture accession is the primary process of imaging processing and it is reported as obtaining the picture through digital device and saves it in electronic basis for extreme MATLAB process. It is additionally a motion of obtaining a photo by using machinery; hence it needs to be surpassed via in addition process. In our work, we collected different hand gestures for MATLAB for handling of depictions.

\section{C.2 Image Pre-processing}

The principal motive of photograph pre-handling is to enhance the picture information involves useless contortions to improve certain picture aspects for in addition handling. Pre-managing technique makes use of various methods such as altering picture dimension and boundaries, denoising, photograph manipulation, improving photo and geological operations. In the current process, we handled-down a range of MATLAB program to alter the dimensions of picture, to decorate distinction and RGB to grayscale transformation. There are many types of filter but right here we used MEDIAN FILTER because to denoise the redundant picture and show values. It does a good method of eliminating redundancy, with reduced dimming of pictures. Thresholding of image is done here by OTSU'S METHOD. The portrayal of picture is threshold so that outline representation is founded.

\section{C.3 Image Segmentation}

Imaging portioning is the process of transformation of binary photo into various segments and furnishing of a picture into something for less difficult process. Handling picture portioning is used for finding the things and outline line of that picture. To partition a picture into known areas with respect to specific usage. It depends on dimensions obtained from picture may be grey level, brightness, consistency, intensity or
Fig. 2. Block diagram of modified stroke rehabilitation

movement. There are many types of segmentation (Graylevel thresholding, Edge detection algorithm, Morphological processing-means clustering method).Here we used edge-detection method for image segmentation process. There are different methods listed out.(CANNY, PREWITT, LOG, ROBERTS, SOBEL, ZEROCROSS).In this CANNY METHOD is chosen because it identifies corners by making use of for local brightness of pixels of intensity of picture. It estimates intensity using cliché of Gaussian filter. It renders 2 thresholds to detect bright and dull corners, including dull corners in results, if they are linked to bright corners. Handling thresholds, canny method is superior to related process to be fooled by redundancy and easily to identify redundant edges.

\section{C.4 Feature Extraction}

Attributes acquiring a method of dimensionality alleviation that correctly notifies fascinating sectors of a photo as a dense function vector. This strategy is beneficial when photo dimensions are massive and a less function identication is needed to shortly whole duties such as picture similarity and obtaining. Attributes identification, characteristic extraction and similarity identification are frequently blended to resolve frequent laptop imaginative and prescient troubles such as thing identification and understanding, information-based picture obtaining, facial features identification, and quality segregation. The random attributes vectors such as brightness, quality, geological characteristics and shape are obtained. Attributes retrieval is process for consisting many of sources need to explain a many information accurately. Quality attributes of any image are processed by Gray level co-occurrence matrix (GLCM) method for quality checking and quality based characteristics are processed from non-dynamical classification of accurate resolution amalgamation at the required space comparative to others. Scale of grey ranges is vital in GLCM and non-dynamicity is labeled into range of primary, secondary \& higher for level of resolution basis in all the amalgamation. Variety of non-dynamical quality attributes of GLCM are level of power, texture analysis, data rate of correlation, entropy, contrast and inverse difference and distinction entropy. Here we used DISCRETE WAVELET TRANFORM BASED FEATURE EXTRACTION METHOD. Wavelet method is an innovative growth place in application mathematics and technological advancements. Due to the better occurrences attributes, wavelet is having many applications. The multiple wavelet basic theoretical process related on multi brightness process. Multiple wavelet method has been an upgraded searched topic in the basis of wavelet. Multiple-wavelet is a derivative from scalar wavelet. In addition to retain the time based attributes, frequency based characteristics and other possessions; it also reduces the pitfalls of the scalar wavelet. Multiple-wavelet has variety of specific possessions such as dimensions, brightness and uniformity that are very essential for the picture processing and implementation. The pictures are segregated into four sub-coefficients or sub-images, by 
application of DWT.These sub-divided bands are named as LL1, LH1, HL1 and HH1. LH1, HL1 and HH1 subcoefficients gives the finest level coefficients, i.e., image based elements and the other band LL1 gives the information about the rough level coefficients i.e., image approximation. To identify the sub divided rough basis characteristics of wavelet coefficients; the sub-divided LL1 can be divided and processed. Relatively, LL2 can be used for other division. This process will occur till the final division. The converted coefficients, photo estimation and picture indispensable points are utilized for quality identification, division and quality attribute retrieval.

\section{C.5 Technology Used (MATLAB)}

MATLAB is a numerical computing environment developed by MathsWork. MATLAB is used because it allows us to do Matrix processing, sketching of values of information and application of coding and obtaining of human being and computer interprocess is easily possible. We use MATLAB Picture manipulation Toolbox, but no toolboxes are required for many process. Instructions are indicated with [Picture Toolbox]. Some of the commands which we have used here are imread \& imwrite which helps MATLAB to identify images respectively. The user can run the MATLAB code to recognize the gestures hence the dexterous 5 finger artificial machine finger can imitate the human gesticulation in current process.

\section{Wavelet Transform Based Feature Extraction}

The wavelet process provides an important identification and better identification for pictures. Wavelet had revealed that it is very required for identification and similarity methods. Multiple brightness method is the major necessary attributes in the wavelet process. The picture is used in de noising with low and high pass filter continued by decimation and it again continue its similar process and it provides four quadrants such as approximation (Low Low Frequency), horizontal (Low High Frequency), Vertical (High Low Frequency) and Diagonal(High High Frequency) Coefficients. The data redundancy is greatly reduced using wavelet transform.

\subsubsection{Selection of Wavelet}

Various types are provided in the wavelet transform. Scholars used various methods to select the wavelet. Entropy related process has been used majorly to choose wavelets. Data retrieval and various error method is required for optimum choosing of wavelet .Based on the characteristics of picture wavelet are choose. There is no individual method or values to select the wavelet.There are different wavelets. In our proposed system we used REVERSE BIORTHOGONAL WAVELET.

\section{2..4.2 Level of Decomposition}

The level of sampling or division of original signal provides the higher formulation of a picture. Depends up on the power of the Sub-coefficients the stage of sampling is terminated. Two stage sampling was done and additional sampling is not needed since the level of power turns to be zero.

\section{FEATURES EXTRACTION}

\section{A. Histogram of Oriented Gradients}

It divides the images into small $(8 * 8)$ pixels and blocks of $(4 * 4)$ cells. Each cell has fixed number of gradient orientation bins. The attributes are provided in a 1 by $\mathrm{N}$ dimension,where $\mathrm{N}$ is a HOG feature length. The obtained attributes gives local dimensions of data from spaces within a picture.

\section{B. Local Binary Pattern}

It returns extracted uniform local binary pattern from a grayscale image, encodes local texture information.

\section{Peak Signal To Noise Ratio}

It returns the peak SNR value and the SNR value based upon the noises built in the image.

\section{Texture Analysis}

There are different methods for texture analysis. Here we used,

\section{I.D.1 Entropy}

It provides a scalar measure comprise the entropy of gray level picture. It is accurate estimate of probability that will be required to distinguish the quality of captured picture.

\section{I.D.2 Entropy Filtering}

Image to be filtered, It returns matrix J,in which each of the resultant smallest element in image consist of entropy measurement of 9 by 9 matrix values over adjacent values in captured picture. It uses symmetric padding.

\section{I.D.3 Range Filtering}

It identifies vertical edges using range filtering. Mainly emphasize vertical edges.

\section{I.D.4 Gray Level Co-occurrence Matrix (GLCM)}

Create gray level co-occurrence array from image. It generates GLCM by estimating the occurrence a value with gray-level (grayscale resolution) value I happens to be present parallel adjoining to smallest element in image with result j.By default graycomatrix measures the GLCM basis on parallel closeness $p$ of the pixels[0 1],it is the picture element in accordance to picture element of on same row.

\section{I.D.5 Mean}

To find the mean of image. The captured picture can be in numbered form/relative expression, the resultant picture is scalar of class double.

\section{I.D.6 Measure Properties of Image Regions}

It produces estimations for set of possessions represented by possessions for every (cc) linked components (things) in cc. The used properties are AREA, PERIMETER, ECCENTRICITY,
Blue Eyes Intelligence Engineering \& Sciences Publication 


\section{ORIENTATION, and SOLIDITY.}

\section{SIMULATION RESULTS}

Different phases of simulation of modified Stroke Rehabilitation System is shown in Fig. $3 \& 4$. Use of median filtering, noise level is reduced. Then, edge detection is achieved with otsu thresholding and canny edge detection algorithm. Final decomposition for movement detection is achieved by DWT process.

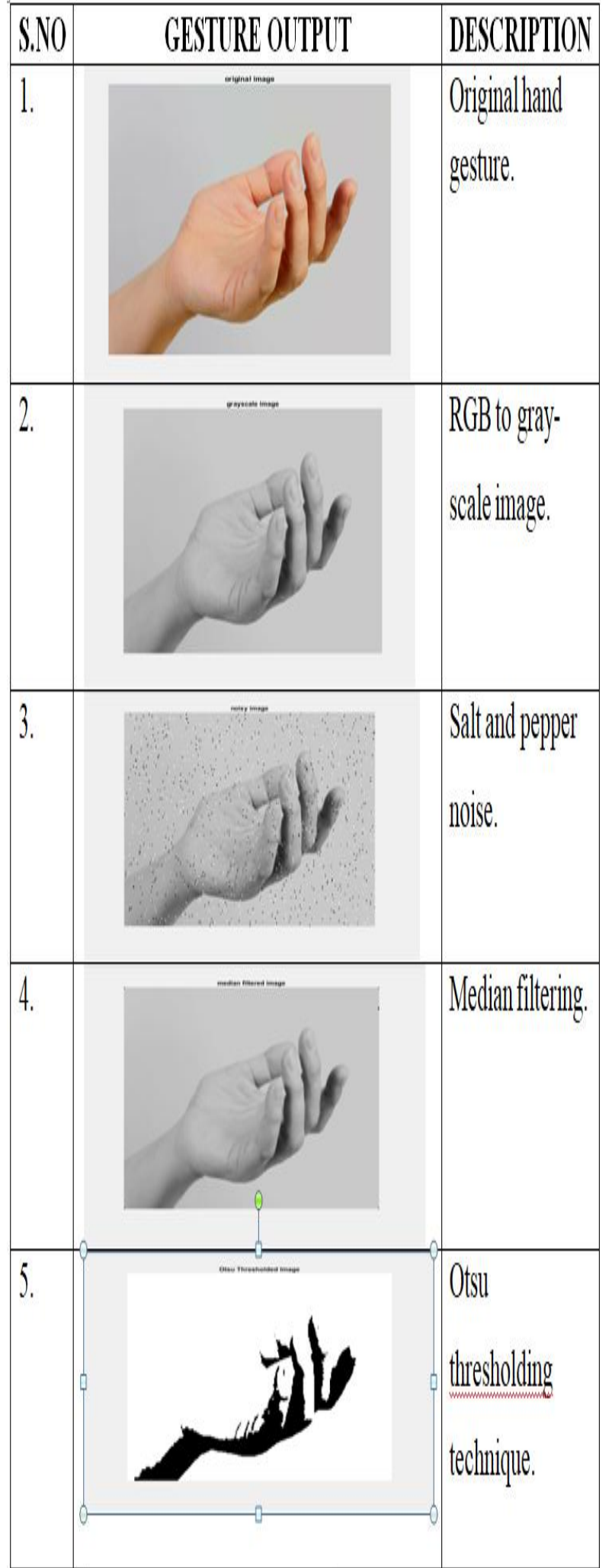

Fig. 3. Simulation results of thresholding techniques in hand gestures

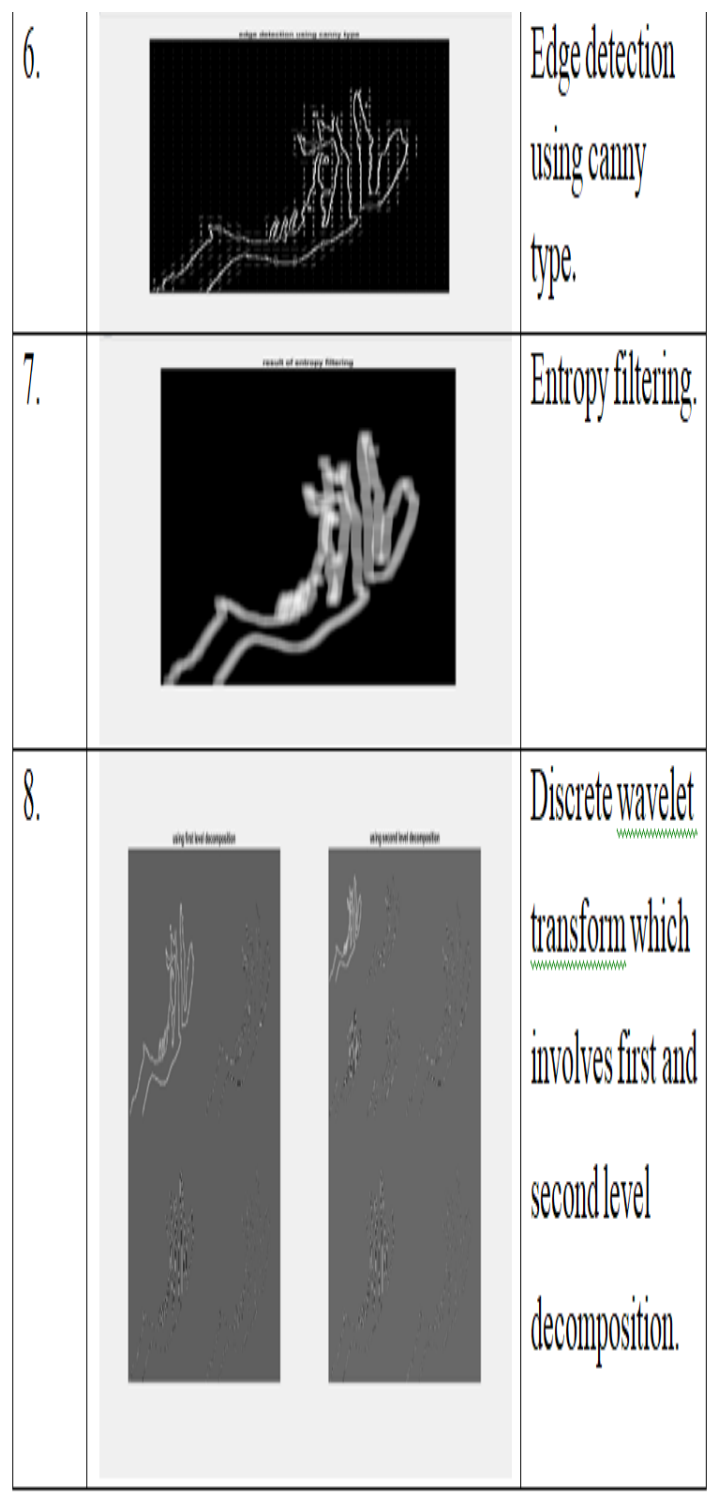

Fig. 4. Simulation results of discrete wavelet transform

\section{USES}

1. Finger gesticulations processed machine for impediment human beings.

2 . Finger gesticulation processed doors and vehicles.

3 . Finger gesticulations controlled keyboard and mouse to make interprocess with machines.

4. Gesticulations processed devices like air conditioner.

5. Growing aids for the deaf people.

6. Accessing very younger adolescents to have interaction along machines.

7. Processing methods for scientific test method.

8. Identifying Gesticulation speech.

9. Hospital based recognizing tension levels.

10. Bluff identification.

11. Gaming applications.

\section{CONCLUSION}

\section{Published By:}

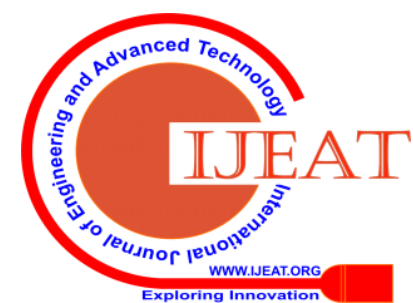


The regular process used to be to render wavelet primarily related to obtaining attributes of hand for segregating hand gestures for stroke rehabilitation system. The notable attributes or traits production was once made via using DISCRETE WAVELET TRANSFORM. Experimental outcomes had proven that higher accuracy outcomes were done through the usage of REVERSE BIORTHOGONAL WAVELET feature sets. Study indicates that when characteristic extraction technique are applied for the image evaluation place based shape function are more sturdy as these techniques extract all the shape information. In this project we have given a thinking of static hand gesture focus with center of attention on the function extraction stage and some of hand gesture focus applications. We have also presented some researches that used distinct hand elements for awareness manner that are make the consciousness process greater accurate. In this project, an important process is suggested to obtain the features of hand gesticulations. The approximate coefficient matrix and small print sub decomposed matrix is received after applying DWT for every hand gesture. From the approximate coefficient matrix, a set of environment friendly statistical characteristic features are obtained to characterize the characteristic of a hand gesture. There are multiple processes to finger gesticulations identification and every method have its power and pitfalls. This file has produced a survey of more than a few finger gesticulation awareness methods. In this paper, the thinking of exceptional processing, Image acquistion, Preprocessing, division, distinguishing attributes based on traits are explained and differentiated on identification and application of finger position and gesticulation processing. This interprocess makes customers to be enabled to process everything in smart way of hand finger gesticulation.

\section{REFERENCES}

1. Anu Maria k Jose, Vigi elezebeth Wilson, mithu susan renji, vijay jose,"Hand gesture controlled robot", International Journal of Scientific \& Engineering Research, Volume 7, Issue 3, March 2016.

2. Durga.s,Gabrielaroselene,m.harshini,m.haneesha,prof.raghavendra mulimani,'Digital image processing based hand gesture recognition robot for multiple applications", International journal of innovative research in electrical, electronics, instrumentation and control engineering, Vol. 4, Issue 5, May 2016.

3. Geng yang et al,"An iot enabled stroke rehabilitation system based on smart wearable armband and machine learning", IEEE, 2017.

4. Kerthana R, Dr.Rajeshkannan S,"A modified BLE enabled stroke rehabilitation system based on machine learning", Fifth international conference on green technologies for power generation, communication instrumentation(ICGPC'19),St.Peter's Institute of higher education and research,Chennai,p. 32,April 2019, unpublished.

5. Kerthana R, Dr.Rajeshkannan S,"A modified zigbee enabled stroke rehabilitation system based on discrete wavelet transform", International Conference on Recent trends in Electronics, Computing and Communication Engineering(ICRTECC'19), Saveetha School

Engineering,SIMATS,Chennai,April 2019,unpublished.

6. Dr.Rajeshkannan S, Kerthana R,"A Modified Digital image processing enabled stroke rehabilitation system based on wavelet transform", Fourth International Conference on Recent Trends in Engineering and Technology(ICRTET-2019),St.joseph's institute of technology,Chennai,pp. 66-71,May 2019,submitted for publication.

7. Rajkumar goel,vineet kumar,saurabh srivastava,a.k.sinha,"A review of feature extraction techniques for image analysis", International Journal of Advanced Research in Computer and
Communication Engineering,Vol. 6, Special Issue 2, February 2017. Sana'a Khudayer Jadwaa et al,'Feature extraction for hand gesture recognition review", International Journal of Scientific \& Engineering Research, Volume 6, Issue 7, July-2015.

8. Shilpa L khandade et al,"MATLAB based gesture recognition", 2016.

9. Soeb hussain, rupal saxena,xie han,jameel ahmed khan,prof.hynchul shin, "Hand gesture recognition using deep learning",IEEE,2017.

\section{AUTHORS PROFILE}

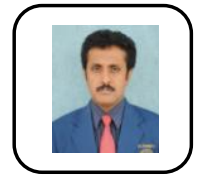

Rajeshkannan Sundararajan received his B.E degree in Electronics and Communication Engineering from Government college of Engineering, Thirunelveli, ManonmaniyamSundaranar University, India in 1998 and M.E in Applied Electronics from Sathyabama University, Chennai, India in 2004. He secured his PhD from Anna University, Chennai in 2016 in the field of computer vision. He is currently working as Associate Professor in the Department of Electronics and communication, St. Joseph's College of Engineering, Chennai, India. He has published more than ten research papers in international journals and many in national and international conferences. He received funded project from Tamilnadu State Council for Science and Technology under student project scheme 2018-19. His research interest includes IoT, image processing and VLSI design.

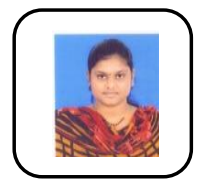

R.kerthanareceived her B.E degree in Electronics and Instrumentation Engineering from Jeppiaar Engineering College, Chennai, India in 2017. She is going to receive the M.E. degree in Embedded system technologies from St.Joseph's college of engineering, Chennai, India in 2019. She has presented her projects in Three International Conferences. Her area of interest includes Embedded systems, Digital Image Processing. 